\title{
Estudo Comparativo entre Fentanil por Vias Peridural e Venosa para Analgesia de Operações Ortopédicas
}

\section{Comparative Study of Epidural and Intravenous Fentanyl for Postoperative Analgesia of Orthopedic Surgeries}

\author{
Marcelo Soares Privado ${ }^{1}$; Rioko Kimiko Sakata, TSA ${ }^{2}$; Adriana Machado Issy ${ }^{2}$; João Batista Santos Garcia, TSA ${ }^{3}$
}

\section{RESUMO}

Privado MS, Sakata RK, Issy AM, Garcia JBS - Estudo Comparativo entre Fentanil por Vias Peridural e Venosa para Analgesia de Operações Ortopédicas

JUSTIFICATIVA E OBJETIVOS: Existem controvérsias sobre o local de ação de opióides lipofílicos após injeção peridural, e alguns autores acreditam que esses fármacos agem no nível supra-espinhal, enquanto outros acham que ocorre ação espinhal. Para tentar esclarecer essa dúvida foi feito estudo comparativo da aplicação de fentanil por vias peridural e venosa após operações ortopédicas de membro inferior.

MÉTODO: O estudo foi aleatório e duplamente encoberto. Quando apresentavam dor pós-operatória, os pacientes do G1 $(n=14)$ receberam $5 \mathrm{ml}$ de solução $(100 \mu \mathrm{g}$ de fentanil em solução fisiológica a 0,9\%) por via peridural e $2 \mathrm{ml}$ de solução fisiológica a 0,9\% por via venosa, os do G2 $(n=15)$ receberam $5 \mathrm{ml}$ de solução fisiológica a $0,9 \%$, por via peridural e $2 \mathrm{ml}$ de fentanil $(100 \mu \mathrm{g})$ por via venosa. Foi avaliada a necessidade de complementação analgésica com tenoxicam (40 mg) por via venosa e com bupivacaína a 0,25\% (5 ml) por via peridural (quando não havia alívio com tenoxicam). A intensidade da dor foi avaliada pelas escalas numérica e verbal nos momentos $M_{30}, M_{120}$ e $M_{240}$ minutos.

RESULTADOS: O número de pacientes que necessitaram de complementação analgésica, tanto com o tenoxicam (G1 = 10 e $G 2=15$ pacientes) quanto com a bupivacaína (G1 = 2 e $G 2=8$ pacientes) foi maior no G2. Não houve diferença estatística na intensidade da dor entre os grupos nos tempos avaliados.

CONCLUSÕES: Nas condições deste estudo o efeito analgésico do fentanil peridural é melhor que por via venosa.

UNITERMOS: ANALGESIA, Pós-Operatória: ANALGÉSICOS, Opióides: fentanil; TÉCNICAS ANESTÉSICAS, Regional: peridural, venosa

\footnotetext{
${ }^{*}$ Recebido do (Received from) Hospital São Paulo, Universidade Federal de São Paulo (UNFESP), São Paulo, SP

1. Pós-Graduando da Disciplina de Anestesiologia, Dor e Terapia Intensiva (UNIFESP)

2. Professora Adjunta e Responsável pelo Setor de Dor da Disciplina de Anestesiologia, Dor e Terapia Intensiva (UNIFESP)

3. Professor Adjunto da Disciplina de Anestesiologia da Universidade Federal do Maranhão
}

Apresentado (Submitted) em 21 de outubro de 2003 Aceito (Accepted) para publicação em 26 de abril de 2004

Endereço para correspondência (Correspondence to) Dra. Rioko Kimiko Sakata

R. Três de Maio 61/51 - Vila Clementino

04044-020 São Paulo, SP

E-mail: riokoks.dcir@epm.br

(C) Sociedade Brasileira de Anestesiologia, 2004

\section{SUMMARY}

Privado MS, Sakata RK, Issy AM, Garcia JBS - Comparative Study of Epidural and Intravenous Fentanyl for Postoperative Analgesia of Orthopedic Surgeries

BACKGROUND AND OBJECTIVES: There are controversies about the action site of lipophylic opioids after epidural injection. Some authors believe that these drugs act at supraspinal level, while others propose a spinal action. This comparative study aimed at answering this question by comparing epidural and intravenous fentanyl for postoperative analgesia of lower limb orthopedic procedures.

METHODS: This was a randomized double-blind study. At postoperative pain complaint, G1 patients $(n=14)$ received $5 \mathrm{~mL}$ epidural solution ( $100 \mu \mathrm{g}$ fentanyl in $0.9 \%$ saline) and $2 \mathrm{~mL}$ of intravenous $0.9 \%$ saline; $G 2$ patients $(n=15)$ received $5 \mathrm{~mL}$ epidural $0.9 \%$ saline and $2 \mathrm{~mL}$ intravenous fentanyl $(100 \mu \mathrm{g})$. Analgesic complementation with intravenous tenoxicam $(40$ $\mathrm{mg}$ ) and epidural $0.25 \%$ bupivacaine $(5 \mathrm{~mL}$ ) (when there was no relief with tenoxicam) has been evaluated. Pain intensity was evaluated by numeric and verbal scales in moments $M_{30}, M_{120}$ and $M_{240}$ minutes.

RESULTS: Number of patients needing analgesic complementation both with tenoxicam (G1 $=10$ and $G 2=15$ patients) and bupivacaine ( $G 1=2$ and $G 2=8$ patients) has been higher in G2. There have been no statistical differences in pain intensity between groups in all studied moments.

CONCLUSIONS: In the conditions of our study, effects of epidural fentanyl were better as compared to intravenous fentanyl.

Key Words: ANALGESIA, Postoperative; ANALGESICS, Opioids: fentanyl; ANESTHETIC TECHNIQUES, Regional: epidural, intravenous

\section{INTRODUÇÃO}

pós a descoberta de receptores localizados na medula Aespinhal e a apresentação de estudo relatando alívio prolongado e intenso da dor após injeção subaracnóidea de morfina ${ }^{1}$, iniciou-se uma nova etapa no tratamento da dor com opióides por via espinhal, tanto para dor aguda como para a crônica.

Um dos opióides mais utilizados por via peridural tem sido o fentanil. Seu início de ação é rápido, com alívio satisfatório da dor e duração de analgesia entre 4 e 8 horas.

As opiniões são controversas em relação ao local de ação do fentanil após injeção peridural. Alguns autores acreditam que seja na medula espinhal, outros, na região supra-espinhal devido a sua alta lipossolubilidade, com conseqüente absorção para a circulação e distribuição para o encéfalo ${ }^{2}$. Alguns estudos atribuem ação sistêmica ao fentanil peridural, pois demonstram que a concentração plasmática encon- 
trada durante os períodos de analgesia está na chamada "faixa analgésica". Entretanto, outros pesquisadores concluem que a ação analgésica do fentanil se dá por ação espinhal ${ }^{3,4}$, pois a concentração plasmática deste medicamento dosado em momentos de conforto e analgesia pós-operatória é inferior à concentração plasmática mínima necessária para analgesia ${ }^{5,6}$. Ainda existem outros autores que acreditam em uma combinação destes dois mecanismos ${ }^{7}$.

A proposta deste estudo foi comparar o efeito analgésico do fentanil por via peridural com a via venosa em pacientes submetidos a operações ortopédicas de membros inferiores.

\section{MÉTODO}

Após a aprovação do estudo pelo Comitê de Ética da Universidade Federal de São Paulo e assinatura do Termo de Consentimento, foram estudados 29 pacientes com idades entre 18 e 65 anos, estado físico ASA I ou II, submetidos a operações ortopédicas de membros inferiores.

O estudo foi aleatório e duplamente encoberto. Os pacientes foram divididos em dois grupos, sendo excluídos do estudo os pacientes com contra-indicação para realização de punção peridural (infecção no local da punção, recusa do paciente, distúrbios da coagulação) e usuários de opióides.

A medicação pré-anestésica administrada nos pacientes foi o diazepam, na dose de $10 \mathrm{mg}$ por via oral, uma hora antes da anestesia.

A anestesia peridural foi realizada através de punção lombar no espaço $L_{3}-L_{4}$ ou $L_{4}-L_{5}$, seguida de injeção de $20 \mathrm{ml}$ de bupivacaína a 0,5\% com adrenalina 1:200.000 e $1 \mathrm{ml}(50 \mu \mathrm{g}) \mathrm{de}$ fentanil. Foi inserido um cateter peridural para suplementação analgésica, caso fosse necessária. A sedação foi realizada com midazolam, após a realização do bloqueio.

No pós-operatório, foi programada analgesia da seguinte maneira: no grupo $1(n=14) 5 \mathrm{ml}$ de solução contendo $100 \mu \mathrm{g}$ de fentanil diluído em solução fisiológica a 0,9\%, por via peridural e 2 ml de solução fisiológica a 0,9\% por via venosa; no grupo $2(n=15) 5 \mathrm{ml}$ de solução fisiológica a $0,9 \%$ por via peridural e $2 \mathrm{ml}$ de fentanil $(100 \mu \mathrm{g})$ por via venosa.

Acomplementação analgésica foi realizada de acordo com a solicitação do paciente, com tenoxicam $(40 \mathrm{mg})$ por via venosa. Se não houvesse alívio da dor seria administrada bupivacaína a $0,25 \%$ (5 ml) por via peridural.

A dor foi avaliada em 30 minutos, 2 e 4 horas após terem sido injetadas as soluções analgésicas, através de escala numérica, de zero a 10 , onde 0 (zero) seria a ausência de dor e 10 (dez) a pior dor possível.

Os resultados obtidos foram submetidos aos testes: Exato deFisher, $t$ de Student, Qui-quadrado e Análise de Variância.

\section{RESULTADOS}

Os dados demográficos (sexo, idade, peso, estatura e índice de massa corpórea) foram semelhantes nos dois grupos (teste Exato de Fisher; teste $t$ de Student) (Tabela I).
Tabela I - Dados Demográficos

\begin{tabular}{lcc}
\hline Parâmetros & Grupo 1 $(\mathrm{n}=14)$ & Grupo 2 $(\mathrm{n}=15)$ \\
\hline Idade $(\mathrm{anos}){ }^{*}$ & $35,28 \pm 15,68$ & $41,26 \pm 13,24$ \\
Peso $(\mathrm{kg})^{*}$ & $71,14 \pm 11,79$ & $74,73 \pm 9,25$ \\
Altura $(\mathrm{cm})^{*}$ & $170,93 \pm 8,27$ & $167,87 \pm 8,32$ \\
Sexo & & \\
$\quad$ Masculino & 11 & 10 \\
$\quad$ Feminino & 3 & 5 \\
$\mathrm{IMC}\left(\mathrm{kg} / \mathrm{m}^{2}\right)^{*}$ & $24,42 \pm 4,12$ & $26,52 \pm 2,46$ \\
\hline
\end{tabular}

não houve significância estatística, $\leq 0,05$; sexo - teste Exato de Fisher; idade, peso, estatura, IMC: teste $t$ Student

* Valores expressos em Média \pm DP

Os procedimentos cirúrgicos realizados foram semelhantes, e não houve diferença significante entre as médias de duração da operação entre os grupos 1 (2h17 minutos) e 2 (2h15 minutos) (Tabela II).

\section{Tabela II - Tipos de Intervenções Cirúrgicas}

\begin{tabular}{lcc}
\hline Tipos de Cirurgias & G1 (n) & G2 (n) \\
\hline Ligamentoplastia de joelho & 7 & 6 \\
Prótese de joelho & 2 & 2 \\
Tratamento cirúrgico de lesão ligamentar de quadríceps & 1 & - \\
Tratamento cirúrgico de lesão ligamentar de quadríceps & 1 & - \\
e osteossíntese de patela & 1 & 1 \\
Osteotomia valgizante de fêmur & 1 & - \\
Revisão de osteotomia & - & 1 \\
Artroscopia de joelho para tratamento de lesão do & & \\
menisco & - & 1 \\
Osteossíntese de patela & 1 & 1 \\
Artrodese modelante do tornozelo e pé & - & 1 \\
Artrodese de tornozelo com haste intramedular & - & 1 \\
Osteossíntese de fratura de tornozelo & - & 1 \\
Osteossíntese de fratura de calcâneo & 14 & 15 \\
Total & &
\end{tabular}

A média do tempo entre o início da anestesia e a administração da solução de fentanil por via peridural (G1) foi de $380 \pm$ 101 minutos e de fentanil por via venosa (G2) de $331 \pm 83 \mathrm{mi}-$ nutos, não apresentando diferença estatística (teste $t$ de Student, $p=0,170)$.

O número de pacientes que necessitaram de complementação analgésica, tanto com o tenoxicam quanto com a bupivacaína, foi maior no grupo 2 (teste Exato de Fisher) (Tabela III).

Tabela III - Necessidade de Complementação Analgésica com Tenoxicam e Bupivacaína durante o Estudo

\begin{tabular}{|c|c|c|}
\hline \multirow{2}{*}{ Grupos } & \multicolumn{2}{|c|}{ Número de Pacientes (\%) } \\
\hline & Tenoxicam & Bupivacaína \\
\hline $\mathrm{G} 1(n=14)$ & $10(71,4)$ & $2(14,3)$ \\
\hline $\mathrm{G} 2(\mathrm{n}=15)$ & $15(100,0)^{*}$ & $8(53,3)^{*}$ \\
\hline
\end{tabular}

* Houve significância estatística - teste Exato de Fisher $(p=0,04$ para o tenoxicam e 0,05 para a bupivacaína) 
A média do tempo entre a injeção de fentanil (G1) e a complementação com o tenoxicam e com a bupivacaína foi semeIhante nos dois grupos (teste de Mann-Whitney, $p=0,2673$ ) (Tabela IV).

Tabela IV - Tempos entre a Injeção de Fentanil por Vias Peridural (G1) ou Venosa (G2) e a Complementação com Tenoxicam e Bupivacaína (Média \pm DP)

\begin{tabular}{lcc}
\hline \multirow{2}{*}{ Grupos } & \multicolumn{2}{c}{ Momentos (min) } \\
\cline { 2 - 3 } & Tenoxicam & Bupivacaína \\
\hline G1 & $125,7 \pm 57,6$ & $135,0 \pm 21,2$ \\
G2 & $98,3 \pm 49,5$ & $161,8 \pm 51,8$ \\
\hline
\end{tabular}

Não houve significância estatística - teste de Mann-Withney Tenoxicam ( $p=0,267)$, bupivacaína $(p=0,711)$

Quando foram comparados os valores referentes à intensidade da dor pela escala numérica nos dois grupos em cada tempo, não houve diferença estatisticamente significativa conforme a tabela V.

Tabela V - Valores da Média e Desvio-Padrão da Escala Numérica nos Dois Grupos em cada Momento (Média \pm DP)

\begin{tabular}{lccc}
\hline & \multicolumn{3}{c}{ Momentos (min) } \\
\cline { 2 - 4 } & $M_{30}$ & $M_{120}$ & $M_{240}$ \\
\hline$G 1(n=14)$ & $0,35 \pm 0,84$ & $2,14 \pm 3,23$ & $2,21 \pm 2,48$ \\
G2 $(n=15)$ & $0,86 \pm 1,68$ & $3,46 \pm 2,74$ & $2,53 \pm 3,11$ \\
\hline
\end{tabular}

Não houve diferença estatisticamente significativa - teste $t$ de Student

\section{DISCUSSÃO}

Muitos autores ${ }^{2,3,8,9-20}$ têm realizado estudos clínicos com o objetivo de esclarecer o mecanismo de ação do fentanil por via peridural. Apesar de muitos deles concluírem que a ação é sistêmica, por absorção para a circulação seguida de efeito encefálico, muitos deles utilizaram infusão continua.

$\mathrm{Na}$ infusão contínua ocorrem concentrações plasmáticas mais elevadas de fentanil, que podem ter ação supra-espi$\mathrm{nhal}^{3}$. As concentrações plasmáticas encontradas nos momentos em que os pacientes apresentavam analgesia estavam próximas ou acima de $0,63 \mathrm{ng} \cdot \mathrm{ml}^{-1}$, considerada como concentração plasmática mínima para eficácia analgésica segundo alguns estudos ${ }^{3,5,6}$. O presente estudo utilizou dose única de fentanil por via peridural e tempo de estudo compatível com a cinética deste opióide.

Apesar da disponibilidade de métodos analíticos adequados para quantificação das concentrações plasmáticas do fentanil, essas não refletem o grau de ligação a receptores opiói- des em locais onde ocorrem os efeitos da droga como a medula espinhal e o encéfalo ${ }^{11}$. No entanto todos os estudos que visam avaliar o local de ação do fentanil por via peridural o fazem desta forma, analisando as concentrações plasmáticas do fentanil, o efeito analgésico e a necessidade de complementação analgésica, pela dificuldade de estudar a ligação desses fármacos aos receptores.

Neste estudo foram avaliados pacientes submetidos a operações semelhantes, para que a intensidade da dor também o fosse.

Os dados demográficos dos grupos foram semelhantes, fato importante para não haver diferença farmacológica.

O tempo de duração das operações assim como o tempo entre o inicio da anestesia e o tempo em que foi administrado o fentanil por via venosa ou peridural no pós-operatório, também foram semelhantes resultando em uma amostra mais homogênea.

O cateter foi colocado o mais próximo possível do dermátomo correspondente ao maior estimulo doloroso, tática também utilizada por outros autores ${ }^{11,21}$, e considerada importante ${ }^{22,23}$. Isto porque proporciona analgesia mais eficaz com menor quantidade de fármacos. Embora muitos profissionais administrem opióide por via peridural, em interespaços vertebrais distantes do correspondente ao dermátomo da incisão cirúrgica, sabe-se que, apesar da difusão mais extensa desses medicamentos em relação aos anestésicos locais, a concentração maior ocorre próxima do local da injeção.

A utilização de um anestésico local associado a um opióide para anestesia peridural foi feita com o objetivo de reduzir a dose de ambas as medicações, de melhorar a qualidade da anestesia e de reduzir a incidência e a intensidade dos efeitos colaterais das medicações utilizadas, que possuem mecanismos de ação diferentes, com analgesia sinérgica $4,6,24,25$

Autilização de fentanil na anestesia peridural poderia interferir na avaliação. Entretanto, é improvável que isso tenha ocorrido, porque após o uso inicial de opióide, foi utilizado o fentanil somente quando o paciente apresentava dor.

Apesar de não ter sido observada diferença de intensidade da dor nos dois grupos, o resultado encontra-se sobre a influência de um maior número de pacientes que utilizaram complementações analgésicas durante o estudo e maior número de pacientes que utilizaram mais de uma complementação analgésica no grupo 2, em que se utilizou fentanil por via venosa.

A necessidade de complementação analgésica foi maior após o uso do fentanil $(100 \mu \mathrm{g})$ por via venosa do que com injeção peridural. 


\section{Comparative Study of Epidural and Intravenous Fentanyl for Postoperative Analgesia of Orthopedic Surgeries}

Marcelo Soares Privado, M.D.; Rioko Kimiko Sakata, TSA, M.D.; Adriana Machado Issy, M.D.; João Batista Santos Garcia, TSA, M.D.

\section{INTRODUCTION}

After the discovery of spinal cord receptors and the publication of a study reporting prolonged and intense pain relief after spinal morphine injection ${ }^{1}$, a new era for the treatment of pain with spinal opioids has started, to control both acute and chronic pain.

Fentanyl has been one of the most used opioid by epidural injection. It has fast onset with satisfactory pain relief and analgesic effects lasting 4 to 8 hours.

There are controversies about fentanyl action site after epidural injection. Some authors believe that it is the spinal cord, while others suggest the supraspinal region due to its high liposolubility, with consequent absorption to the circulation and distribution to brain ${ }^{2}$.

Some studies attribute systemic action to epidural fentanyl, because they have shown that plasma concentrations during analgesia are within the so-called "analgesic range". However, other investigators have concluded that fentanyl analgesic action is by spinal action ${ }^{3,4}$, because plasma concentration of this drug during moments of comfort and postoperative analgesia is lower as compared to minimum plasma concentration needed for analgesia ${ }^{5,6}$. Still others believe in a combination between both mechanisms ${ }^{7}$.

This study aimed at comparing epidural and intravenous fentanyl for postoperative analgesia of lower limb orthopedic procedures.

\section{METHODS}

After the Ethics Committee, Unversidade Federal de São Paulo approval and their informed consent, participated in this randomized double-blind study 29 patients aged 18 to 65 years, physical status ASA I or II, submitted to lower limb orthopedic procedures.

Patients were divided in two groups and exclusion criteria were epidural puncture counterindication (puncture site infection, patients' refusal, coagulation disorders) and opioid users.

Patients were premedicated with $10 \mathrm{mg}$ oral midazolam, one hour before surgery.

Epidural anesthesia was induced with lumbar puncture at $L_{3}-L_{4}$ or $L_{4}-L_{5}$ interspace, followed by $20 \mathrm{~mL}$ of $0.5 \%$ bupivacaine with 1:200,000 epinephrine and $1 \mathrm{~mL}(50 \mu \mathrm{g}$ fentanyl). Epidural catheter was inserted for analgesic complementation, if needed. Sedation was induced with midazolam, after blockade.
Postoperative analgesia was scheduled as follows: group 1 ( $n=14$ ) would receive $5 \mathrm{~mL}$ epidural solution with $100 \mu \mathrm{g}$ fentanyl diluted in $0.9 \%$ saline and $2 \mathrm{~mL}$ intravenous $0.9 \%$ saline; group $2(n=15)$ would receive $5 \mathrm{~mL}$ epidural $0.9 \%$ saline and $2 \mathrm{~mL}$ intravenous fentanyl $(100 \mu \mathrm{g})$.

Analgesia would be complemented according to patient's request, with intravenous tenoxicam $(40 \mathrm{mg})$, and epidural $0.25 \%$ bupivacaine $(5 \mathrm{~mL})$ in case of no pain relief.

Pain was evaluated at 30 minutes, 2 and 4 hours after analgesic solutions injection, by numeric scale from zero to 10 , where zero (0) was no pain and ten (10) the worst imaginable pain.

Results were submitted to Fisher Exact test, Student's $t$ test, Chi-square test and Analysis of Variance.

\section{RESULTS}

Demographics (gender, age, weight, height and body mass index) were similar between groups (Fisher Exact test; Student's $t$ test) (Table I).

Table I - Demographics Data

\begin{tabular}{lcc}
\hline Parameters & Group 1 $(\mathrm{n}=14)$ & Group 2 $(\mathrm{n}=15)$ \\
\hline Age $(\text { years })^{*}$ & $35.28 \pm 15.68$ & $41.26 \pm 13.24$ \\
Weight $(\mathrm{kg})^{*}$ & $71.14 \pm 11.79$ & $74.73 \pm 9.25$ \\
Height $(\mathrm{cm}){ }^{*}$ & $170.93 \pm 8.27$ & $167.87 \pm 8.32$ \\
Gender & & \\
$\quad$ Male & 11 & 10 \\
$\quad$ Female & 3 & 5 \\
BMI $\left(\mathrm{kg} / \mathrm{m}^{2}\right)^{*}$ & $24.42 \pm 4.12$ & $26.52 \pm 2.46$ \\
\hline
\end{tabular}

There has been no statistical significance, $\leq 0.05$; gender - Fisher Exact test; age, weight, height, BMI: Student's $t$ test

* Values expressed in Mean \pm SD

Surgical procedures were similar without significant differences in surgery mean length for group 1 (137 minutes) and group 2 (135 minutes) (Table II).

Table II - Surgeries

\begin{tabular}{lcc}
\hline Types & G1 (n) & G2 (n) \\
\hline Knee Ligament Repair & 7 & 6 \\
Knee replacement & 2 & 2 \\
Surgical quadriceps ligament injury repair & 1 & - \\
Surgical quadriceps ligament injury repair and & 1 & - \\
patellar osteosynthesis & 1 & 1 \\
Femoral valgizing osteotomy & 1 & - \\
Osteotomy review & - & 1 \\
Knee arthroscopy for meniscus repair & - & 1 \\
Patellar osteosynthesis & 1 & 1 \\
Ankle and foot modeling arthrodesis & - & 1 \\
Ankle arthrodesis with intramedullary rod & - & 1 \\
Fractured ankle osteosynthesis & - & 1 \\
Fractured heel osteosynthesis & 14 & 15 \\
Total &
\end{tabular}


Mean time between beginning of anesthesia and epidural fentanyl injection (G1) was $380 \pm 101$ minutes and between beginning of anesthesia and intravenous fentanyl injection (G2) was $331 \pm 83$ minutes, without statistical difference (Student's $t$ test, $\mathrm{p}=0.170)$.

Number of patients needing analgesic complementation both with tenoxicam and bupivacaine has been higher in Group 2 (Fisher Exact test) (Table III).

Table III - Need for Analgesic Complementation with Tenoxicam and Bupivacaine during the Study.

\begin{tabular}{lcc}
\hline \multirow{2}{*}{ Groups } & \multicolumn{2}{c}{ Number of Patients (\%) } \\
\cline { 2 - 3 } G1 $(n=14)$ & Tenoxicam & Bupivacaine \\
$\mathrm{G} 2(\mathrm{n}=15)$ & $10(71.4)$ & $2(14.3)$ \\
\hline
\end{tabular}

* There has been statistical significance - Fisher Exact test ( $p=0.04$ for tenoxicam and 0.05 for bupivacaine)

Mean time between fentanyl injection and tenoxicam and bupivacaine complementation has been similar for both groups (Mann-Whitney test, $\mathrm{p}=0.2673$ ) (Table IV).

Table IV - Times Between Epidural (G1) or Intravenous (G2) Fentanyl Injection and Complementation with Tenoxicam and Bupivacaine (Mean \pm SD)

\begin{tabular}{lcc}
\hline \multirow{2}{*}{ Groups } & \multicolumn{2}{c}{ Moments $(\min )$} \\
\cline { 2 - 3 } & Tenoxicam & Bupivacaine \\
\hline G1 & $125.7 \pm 57.6$ & $135.0 \pm 21.2$ \\
G2 & $98.3 \pm 49.5$ & $161.8 \pm 51.8$ \\
\hline
\end{tabular}

There has been no statistical significance - Mann-Withney test Tenoxicam $(p=0.267)$, bupivacaine $(p=0.711)$

Pain intensity evaluated by numeric scale for both groups in each moment has not shown statistically significant differences, as shown in table $\mathrm{V}$.

Table V - Mean and Standard Deviation of Numeric Scale for Both Groups in each Moment (Mean \pm SD)

\begin{tabular}{lccc}
\hline & \multicolumn{3}{c}{ Moments (min) } \\
\cline { 2 - 4 } & $M_{30}$ & $M_{120}$ & $M_{240}$ \\
\hline$G 1(n=14)$ & $0.35 \pm 0.84$ & $2.14 \pm 3.23$ & $2.21 \pm 2.48$ \\
$\mathrm{G} 2(n=15)$ & $0.86 \pm 1.68$ & $3.46 \pm 2.74$ & $2.53 \pm 3.11$ \\
\hline
\end{tabular}

There has been no statistically significant difference - Student's $t$ test

\section{DISCUSSION}

There are several clinical studies aiming at explaining the action mechanism of epidural fentanyl ${ }^{2,3,8,9-20}$. Although many have concluded that the action is systemic, by absorption to circulation followed by cerebral effect, some have used continuous infusion.

Continuous infusion leads to higher plasma fentanyl concentrations, which may have supraspinal action ${ }^{3}$. Plasma concentrations found when patients presented analgesia were close to or above $0.63 \mathrm{ng} \cdot \mathrm{mL}^{-1}$, considered by some studies as minimum plasma concentration for analgesic efficacy ${ }^{3,5,6}$.
Our study has used fixed epidural fentanyl dose and timing compatible with this opioid kinetics.

In spite of the availability of adequate analytical methods to quantify fentanyl plasma concentrations, these do not reflect the level of binding of opioids in sites of drug effects, such as spinal cord and brain ${ }^{11}$. However, all studies aiming at evaluating epidural fentanyl action site do it this way, analyzing fentanyl plasma concentrations, analgesic effect and need for analgesic complementation, due to the difficulty in studying the binding of those drugs to receptors.

Our study has evaluated patients submitted to similar surgical procedures to compare similar pain intensities.

Demographics data were similar between groups, which is an important factor to prevent pharmacological differences.

Surgery length, as well as time between beginning of anesthesia and postoperative intravenous or epidural fentanyl were also similar, resulting in a more homogeneous sample.

Catheter was placed as close as possible to the dermatome corresponding to higher painful stimulation, tactic also adopted by other authors ${ }^{11,21}$ and considered important ${ }^{22,23}$ because it allows more effective analgesia with less drugs. Although many professionals administer epidural opioids in vertebral interspaces apart from those corresponding to surgical incision dermatome, it is known that, in spite of more extensive spread of these drugs as compared to local anesthetics, higher concentration is seen close to injection site.

Local anesthetics associated to opioid for epidural anesthesia aimed at decreasing both drugs doses, improving anesthesia and decreasing the intensity of side effects of the drugs, which have different action mechanisms with synergistic analgesia ${ }^{4,6,24,25}$.

Fentanyl for epidural anesthesia could have interfered with the evaluation. However it is unlikely that this may have happened because after initial opioid use, fentanyl has only been used when patients referred pain.

Although the absence of difference in pain intensity in both groups, results have been influenced by a larger number of patients using analgesic complementation throughout the study and a large number of patients needing more than one analgesic complementation in group 2, where intravenous fentanyl was used.

The need for analgesic complementation has been higher after intravenous fentanyl $(100 \mu \mathrm{g})$ as compared to epidural injection.

\section{REFERÊNCIAS - REFERENCES}

01. Wang JK, Nauss LA, Thomas JE - Pain relief by intrathecally applied morphine in man. Anesthesiology, 1979;50:149-151.

02. Sandler AN, Stringer D, Panos L et al - A randomized, double-blind comparison of lumbar epidural and intravenous fentanyl infusions for postthoracotomy pain relief. Analgesic, pharmacokinetic, and respiratory effects. Anesthesiology, 1992;77:626-634.

03. Coda BA, Brown MC, Schaffer R et al - Pharmacology of epidural fentanyl, alfentanil, and sufentanil in volunteers. Anesthesiology, 1994;81:1149-1161. 
04. D'Angelo R, Gerancher JC, Eisenach JC et al - Epidural fentanyl produces labor analgesia by a spinal mechanism. Anesthesiology, 1998;88:1519-1523.

05. Gourlay GK, Kowalski SR, Plummer JL et al - Fentanyl blood concentration-analgesic response relationship in the treatment of postoperative pain. Anesth Analg, 1988;67:329-337.

06. de Leon-Casasola OA, Lema MJ - Postoperative epidural opioid analgesia: what are the choices? Anesth Analg, 1996;83: 867-875.

07. Thomson CA, Becker DR, Messick Jr JM et al - Analgesia after thoracotomy: effects of epidural fentanyl concentration/ infusion rate. Anesth Analg, 1995;81:973-981.

08. Justins DM, Francis D, Houlton PG et al - A controlled trial of extradural fentanyl in labour. Br J Anaesth, 1982;54:409-414.

09. Reynolds F, O Sullivan G - Epidural fentanyl and perineal pain in labour. Anaesthesia, 1989;44:341-344.

10. Renaud B, Brichant JF, Clergue $\mathrm{F}$ et al - Ventilatory effects of continuous epidural infusion of fentanyl. Anesth Analg, 1988;67:971-975.

11. Ellis DJ, Millar WL, Reisner LS - A randomized double-blind comparison of epidural versus intravenous fentanyl infusion for analgesia after cesarean section. Anesthesiology, 1990;72: 981-986.

12. Loper KA, Ready LB, Downey MP et al - Epidural and intravenous fentanyl infusions are clinically equivalent following knee surgery. Anesth Analg, 1990;70:72-75.

13. Salomaki TE, Laitinen JO, Nuutinen LS - A randomized double-blind comparison of epidural versus intravenous fentanyl infusion for analgesia after thoracotomy. Anesthesiology, 1991;75:790-795.

14. Welchew EA, Thornton JA - Continuous thoracic epidural fentanyl. A comparison of epidural fentanyl with intramuscular papaveretum for postoperative pain. Anaesthesia, 1982;37: 309-316.

15. Guinard J, Mavrocordatos $P$, Chiolero $R$ et al - A randomized comparison of intravenous versus lumbar and thoracic epidural fentanyl for analgesia after thoracotomy. Anesthesiology, 1992;77:1108-1115.

16. Baxter AD, Laganiere S, Samson B et al - A comparison of lumbar epidural and intravenous fentanyl infusions for post-thoracotomy analgesia. Can J Anaesth, 1994;41:184-191.

17. Cooper DW, Ryall DM, Desira WR - Extradural fentanyl for postoperative analgesia: predominant spinal or systemic action? $\mathrm{Br}$ J Anaesth, 1995:74:184-187.

18. Harukuni I, Yamaguchi $\mathrm{H}$, Sato $\mathrm{S}$ et al - The comparison of epidural fentanyl, epidural lidocaine, and intravenous fentanyl in patients undergoing gastrectomy. Anesth Analg, 1995;81: 1169-1174.

19. Liu SS, Gerancher JC, Bainton BG et al - The effect of electrical stimulation at different frequencies on perception and pain in human volunteers: epidural versus intravenous administration of fentanyl. Anesth Analg, 1996;82:98-102.
20. Lutti MN, Simoni RF, Cangiani LM et al - Analgesia controlada pelo paciente com morfina ou fentanil no pós-operatório de reconstrução de ligamentos do joelho: estudo comparativo. Rev Bras Anestesiol, 2000;50:8-13.

21. Naulty JS, Datta S, Ostheimer GW et al - Epidural fentanyl for postcesarean delivery pain management. Anesthesiology, 1985;63:694-698.

22. Birnbach DJ, Johnson MD, Arcario T et al - Effect of diluent volume on analgesia produced by epidural fentanyl. Anesth Analg, 1989;68:808-810.

23. Dickenson AH, Sullivan AF, McQuay HJ - Intrathecal etorphine, fentanyl and buprenorphine on spinal neurones in the rat. Pain, 1990;42:227-234.

24. Maves TJ, Gebhart GF - Antinociceptive synergy between intrathecal morphine and lidocaine during visceral and somatic nociception in the rat. Anesthesiology, 1992;76:91-99.

25. Penning JP, Yaksh TL - Interaction of intrathecal morphine with bupivacaine and lidocaine in the rat. Anesthesiology, 1992;77: 1186-1200.

\section{RESUMEN}

Privado MS, Sakata RK, Issy AM, Garcia JBS - Estudio Comparativo entre Fentanil por Vías Peridural y Venosa para Analgesia de Operaciones Ortopédicas

JUSTIFICATIVA Y OBJETIVOS: Existen controversias sobre el local de acción de opioides lipofílicos después de inyección peridural, y algunos autores acreditan que eses fármacos actúan en el nivel supra-espinal, en cuanto otros suponen que ocurre acción espinal. Para tentar esclarecer esa duda fue hecho estudio comparativo de la aplicación de fentanil por vías peridural y venosa después de operaciones ortopédicas de miembro inferior.

MÉTODO: El estudio fue aleatorio y duplamente encubierto. Cuando presentaban dolor pos-operatorio, los pacientes del G1 $(n=14)$ recibieron $5 \mathrm{ml}$ de solución $(100 \mu \mathrm{g}$ de fentanil en solución fisiológica a 0,9\%) por vía peridural y $2 \mathrm{ml}$ de solución fisiológica a $0,9 \%$ por vía venosa, los del G2 $(n=15)$ recibieron $5 \mathrm{ml}$ de solución fisiológica a $0,9 \%$, por vía peridural y $2 \mathrm{ml}$ de fentanil $(100 \mu \mathrm{g})$ por vía venosa. Fue evaluada la necesidad de complementación analgésica con tenoxicam $(40 \mathrm{mg})$ por vía venosa y con bupivacaína a 0,25\% (5 ml) por vía peridural (cuando no había alivio con tenoxicam). La intensidad del dolor fue evaluada por las escalas numérica y verbal en los momentos $M_{30}, M_{120}$ y $M_{240}$ minutos.

RESULTADOS: El número de pacientes que necesitaron de complementación analgésica, tanto con el tenoxicam (G1 = 10 y $G 2=15$ pacientes) cuanto con la bupivacaína (G1 $=2$ y $G 2=8$ pacientes) fue mayor en el G2. No hubo diferencia estadística en la intensidad del dolor entre los grupos en los tiempos evaluados.

CONCLUSIONES: En las condiciones de este estudio el efecto analgésico del fentanil peridural es mejor que por vía venosa. 